\title{
The role of errorful and errorless training on error detection and learning of tracking task
}

\author{
Ehsan ZAREIAN, Majid ARAB, Karen BARANIE, Mehdi DASTFAL
}

Department of Motor Behavior, Allameh tabataba'i University, Tehran, Iran.

Address Correspondence to E. Zareian, ehsan.zarian@gmail.com

\begin{abstract}
The aim of this study is to investigate errorful and errorless training methods on detecting error in tracking task. 24 students of Allameh Tabataba'i University ( $23 \pm 2.8$ yrs.) were randomly selected to perform a computerized tracking test. The participants underwent a pre-test after being given verbal instructions and were divided into two groups of errorful and errorless training according to the test results. The errorless training group, during task performance, saw the target path and the path they were tracking as a diagram and detected errors according to the difference between the two paths. But the errorful training group completed the task goal by trial-and-error. Each group underwent 30 tests in 3 separate sessions with a recovery period of $24 \mathrm{~h}$ inbetween. The results showed the significant effect of errorful and errorless training on the progress of detecting errors in acquisition and retention stages of tracking task. Furthermore, a significant difference has been identified between the effects of errorful and errorless training methods on the acquisition and retention of tracking task. This difference was in favor of the errorless training group and this group's progress in detecting errors was more than the errorful training group. The results showed that in addition to the influence of errorful and errorless training methods on detecting error in tracking task, there is a difference between the effect of these methods in detecting error and the errorless training method has a more positive effect on participants' error detecting ability in the tracking task.
\end{abstract}

Keywords: Error detection, errorful training, errorless training, learning, tracking task.

\section{INTRODUCTION}

Motor learning is rather permanent changes in motor behavior which is gained through experience or training and leads to rather sustainable changes in the ability to perform tasks professionally (13). Making a person more capable of assessing this motor behavior is one benefit of training. This means that by training, the subject improves the ability to detect errors with which he can replace augmented feedback and identify the error in his behavior in this way. In fact, the ability to detect errors is a type of ability to utilize internal feedback information for detecting the amount of motor error. In other words, the learned ability to detect errors which is gained through the analysis of feedback from the response is called error detection capability. The general procedure for obtaining error detection capability is being sensitive to the response feedback (15).

Another subject is the methods used in learning the movements. Humans acquire new motor skills during multi-stage learning process which is based on training. The errorful and errorless training methods are two of these processes. In the errorful training method, the participant obtains skill learning through trial and error method. Avoiding errors during this type of training is not possible. In the errorless training method, learning through guidance and feedback methods prevents creating errors in the participant's training. The trial and error method is one of the errorful training methods. Participants correct the motor skills and improve their performance through the trial and error method (8). Prather observed that subjects who trained under errorless conditions had acquired temporary skill improvement which was similar to the implicit and U-mode learning strategy. Subjects learning under trial and error conditions seemed to actively test hypotheses, similar to explicit or S-mode learning. Studies have shown that when the errors are eliminated or significantly reduced during learning, an un-selective learning strategy is created. But when there are errors present during learning, 
which must be corrected, a selective learning strategy is formed (10). The errorful subjects are expected to adapt to correcting their errors during learning with an obvious hypothesis test strategy which is characterized by verbal rules and the subject's reduced performance under the dual task condition. It is estimated that the reduction of errors during learning causes the minimization of the obvious hypothesis test by the subject which leads to the subject's adaptation with an unselective learning strategy which is characterized by less verbal rules and stable performance under the dual task condition. Various studies have supported these results. The golf skill performance in errorless subjects was not affected under the dual task condition, whereas the errorful subjects' performance was ruined under the dual task condition. The error reduction during learning limits the amount of the error correction hypothesis test which causes the reduction of the obvious processing association in skill acquisition. It was concluded that error reduction during learning directs the subject to use unselective and implicit learning processes which causes the subject to be unprepared for performance at times of distraction (10). The results of Feygin et al. (7) showed that sensory guidance is effective to learn timing. It was also found that sensory guidance can be useful for performance, especially while training for the temporal aspects of a task. Furthermore, the results revealed that timing is one of the important aspects of transition from training to the learning environment. Sensory guidance can be helpful in the transition training.

There have been various studies in the field of error detection in various skills through different training methods. Azrati et al. (2) did a research on the effect of complementary special practice with variable practices and mental estimation of error on the deviation, stability and the movement error detection capability in shooting task. Finally, the retention and transfer tests results support the fact that complementary special practice with variable practices in the acquisition state, strengthen the error detection capability and may be a good explanation to consider complementary special practice with variable practice as a good training method (2). Latest studies results like Williams, Mark \& Hodges (19) suggested that observation and feedback interaction helps in both shaping and refining a new movement because the subjects learn what to do by observing the model. A correction reference is formed based on this and when the feedback is presented to the subject, the related feedback is compared with the correction reference and skill refinement takes place gradually and the error detection capability develops (18). Blandin \& Proteau (3) did a research on the cognitive basis of observational learning and the development of mechanisms for the detection and correction of errors. Black et al. (4) investigated the effect of observational practice on error detection and executing movement. The results showed that observational practice was useful in detection of error and execution of movement. Black et al. (4) did a research on learning to detect error in movement timing using physical and observational practice. The results revealed that observers and physical practice participants estimated the durations of movement sequences more accurately than those who only observed the timing task and had no practice. These data provided evidence that recognizing error in movement timing can be accomplished via observation and that observational practice can facilitate acquisition of movement behaviors and improve error detection. Abbas zade et al. (1) did a research on the effects of various error estimation methods and reduced frequency of augmented feedback on the error detection capability, performance and bimanual coordination task learning. The results showed no significant difference in the error estimation and augmented feedback frequency regarding the error estimation accuracy in the acquisition stage. Furthermore, the model error estimation and combination error estimation group were better in the movement timing performance than the other groups. In the retention stage, all the three error estimation groups performed better than the control group regarding model learning and movement timing. And the $100 \%$ model error estimation group and the $100 \%$ timing error estimation group both developed better learning regarding the movement timing than the other groups. Shafizadeh \& Bahram (17) estimated the effect of auditory model with respect to the role of feedback frequency and the task difficulty on learning and error detection of a segmental-timing task. The results revealed that the learning rate in auditory model and feedback groups were better than the groups without auditory model and feedback regarding the relative timing. Regarding the absolute timing, the reduction of the auditory model frequency and feedback presentation leads to better learning and recognizing. There was also a significant mutual effect regarding 
auditory model and task difficulty so that the group with $100 \%$ easy-model and no difficult-model developed a better learning in relative timing. Sabzi \& Mohammadi (14) did a research on the effect of selfcontrol feedback and reduction of knowledge of results frequency on the error detection capability. The results of the acquisition and retention stages revealed that the self-control group with $100 \%$ feedback frequency performed better than the other groups. The self-control group also performed better than the others in the transition/transfer stage. The research results reveal that due to its benefits, giving the subjects the right to choose to receive feedback causes increase in the error detection capability rather than reduced knowledge of results frequency. It can also be noted that the subject-oriented feedback planning is more effective than the instructor-oriented plan. Pitel et al. (12) showed that Using errorless learning, alcoholics improved their abilities to learn new concepts' labels. Moreover new knowledge acquired with errorless learning was flexible. The errorless learning advantage may rely on explicit rather than implicit memory processes in these alcohol-dependent patients presenting only mild to moderate deficits of explicit memory capacities. Jones et al. (9) showed that during the mazes transfer task, the trial-and-error group performed significantly better than the errorless group when there was a requirement to transfer the skill learned during the acquisition phase to a new related task. Schmitz et al. (16) showed an advantage for the errorless condition of procedural skill acquisition, in the Alzheimer's disease. De Werd et al. (6) showed that errorless learning is more effective in teaching adults with dementia a variety of meaningful daily tasks or skills, with gains being generally maintained at follow-up. Ownsworth et al. (11) showed that error-based learning has the potential to reduce the length and costs of rehabilitation and lifestyle support because the techniques could enhance generalization success and lifelong application of strategies after traumatic brain injury.

However, in spite of all the research done in the field of motor skills error detection and the difference between the effects of errorful and errorless training methods on the error detection capability in perceptual motor skills, further research is needed. The present research, with the general belief that errorful and errorless training methods affect the error detection capability in tracking task, aims to investigate the effect of each training method and the differences in subjects; error detection development and error detection capability in tracking task. The results of this research can be used in educating the subjects in motor and athletic tasks which require error detection and correction.

\section{MATERIAL \& METHOD}

The research method was of semi-experiential type. Statistical community of the research was composed of M.A students of Allameh Tabataba'i University. A sample of 24 persons was chosen randomly and simply from this community. Features included eye sight and complete physical health, having complete satisfaction to participate in the research, right hand superiority and age range of $23 \pm$ 2.8 years. The used tool in this research was a laptop that had two hardware and software parts. Software part of the device was a computerized tracking test that has been designed by Borland Delphi 7 programming software. In this assignment the participants should go through the given tracking path. The movement total time period was $10.40 \mathrm{~s}$. At first the superior hand of the participants was determined by the Briggs \& Nebes hand superiority questionnaire (6) and 24 persons with right hand superiority were chosen as sample participants. Before performing the test practically, suitable verbal instruction was given to the participants. The method of the task execution was in this form that the participants executed the given tracking test and entered the number of the time spent on the test into the program and the program showed the percent of their errors. At first, all the participants underwent a pretest and were placed into two similar errorful and errorless training groups according to the pretest results. Each group underwent 30 tests in 3 separate sessions with a recovery period of $24 \mathrm{~h}$ in-between. The designed tasks had the following differences for both errorful and errorless training groups: The errorless training group saw the given tracking path and the path that they tracked themselves as a diagram drawn by the program and identified error at the end of the test; but the errorful training group could see neither the target path, nor the path that they tracked while performing the task and identified error without having any feedback. At end of each training session, an acquisition test was taken from the participants. Finally, error detection retention tests were taken from the participants 24 and 48 hours after the last training session. 


\section{Statistical Analysis}

To describe data, descriptive statistics was used and to analyze statistical hypotheses, deductive statistics was used including the KolmogorovSmirnov statistical test (to investigate normality of data), Levene's statistical test (to investigate homogeneity of variances) and repeated measure ANOVA. All statistical operation was done by SPSS 18 software.

To assess the effect of errorful and errorless training methods on retention of error detection in tracking task, the error detection data gathered from the participants of the two errorful and errorless training groups in the acquisition and retention tests were analyzed 24 and 48 hours after the last training session.

\section{RESULTS}

After normality of data and homogeneity of variances were confirmed, the within-subject effects table showed the following results:

Table 1 . The within-subject effects table.

\begin{tabular}{lcccc}
\hline Test & Sig & F & df & $\eta^{2} \mathrm{p}$ \\
\hline Within-subject effects & $0.001 *$ & 14.60 & 2 & 0.39
\end{tabular}

* The significance level: $\mathrm{P} \leq 0.05$

At the 0.05 significance level, the results of the table showed the significant effect of errorful and errorless learning method on retention of error detection in perceptual-motor skill of the participants. Bonferroni Post hoc test showed the following results:

Table 2. The results of the paired comparison of different stages.

\begin{tabular}{lccc}
\hline Test & Mean difference & S.D & Sig \\
\hline & & & \\
Posttest- 24hr retention & -7.47 & 1.63 & 0.001 * \\
Posttest- 48hr retention & -8.10 & 1.80 & 0.001 * \\
& & & \\
\hline * The significance level: $\mathrm{P} \leq 0.05$ & & &
\end{tabular}

The results of the above table showed the significant effect of errorful and errorless learning method on the development of error detection in perceptual-motor skill during retention stages after 24 and $48 \mathrm{~h}$. The table of inter-group effects showed the following results:
Table 3. The inter-group comparison (between errorful and errorless groups).

\begin{tabular}{lcccccc}
\hline Source & $\begin{array}{c}\text { Sum of } \\
\text { Squares }\end{array}$ & df & $\begin{array}{c}\text { Mean } \\
\text { square }\end{array}$ & F & $p$ & $\eta^{2} p$ \\
\hline Group & 2017.09 & 1 & 2343.50 & 21.60 & $0.001^{*}$ & 0.29 \\
Error & 2053.74 & 22 & 93.35 & & &
\end{tabular}

* The significance level: $\mathrm{P} \leq 0.05$

At the 0.05 significance level, the obtained results showed a significant difference between the errorful and the errorless training group. The diagram of the participants' error detection development during acquisition and retention stages is shown in the following image.

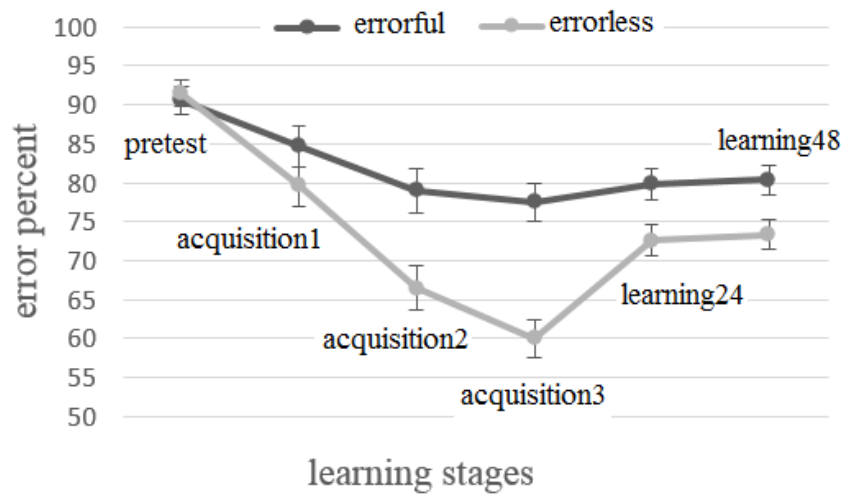

Figure 1. Subjects scores in learning stages.

The results obtained from the diagram show that firstly, there is a difference between both groups. Also, it is clear that the errorless group has had more development in error detection and more reduction in the error percentage.

\section{DISCUSSION}

The aim of the present research was to investigate the effect of errorful and errorless training methods on error detection of perceptual-motor skill. According to the information obtained from the within-subjects effects table, by taking the 0.05 significance level into consideration, the errorful and errorless training methods both have had a significant effect on retention of error detection of perceptual-motor skill. In the 0.05 significance level, with respect to the intergroup effects table, a significant difference was seen between the methods of both errorful and errorless training 
groups and the results revealed that the errorless group showed more improvement in error detection and that their error percentage has reduced more during retention test. Simultaneously augmented feedback was used for performing the errorless training method which included displaying the target path and the participant's executed path during the task and so the significant effect of the errorless training method on the retention and the development of the participants' error detection capability was revealed. The obtained results for the development and retention of error detection are aligned with the researches of Azrati et al. (2) in the field of effect of complementary special practice with variable practice on identification of error, Williams, Mark \& Hodges (19) in the field of the effect of interaction of observation and feedback (as errorless methods), Blandin \& Proteau (4) in the field of effect of observation on identification of error, Black et al. (5) about the effect of observation on identification of error, Shafizadeh \& Bahram (17) which all showed that feedback causes more error detection capability; and the research of Abbas zade et al. (1) which showed that feedback presentation causes improvement in retention of error detection; and also the research of Sabzi \& Mohammadi (14) that showed the groups with a $100 \%$ feedback are better in acquisition and retention than the groups with less feedback. However, the obtained results are not aligned with the research of Abbas (1) that showed that different feedback frequencies have no significant difference in the error detection acquisition stage.

The fact that errorless training method causes a significant improvement on acquisition and retention of error detection is consistent with theoretical foundations, too. According to Adams' theory, the total movements are done by the comparison of the feedback from the movement during performance and the perceptual rejection. When a person does a movement, this movement causes an internal feedback and an augmented feedback. The internal feedback in fact forms a trace on the nervous system. Since a person receives augmented feedback in movements, he/she gets closer to the goal after several efforts, and these correct movements cause the formation of stronger correct perceptual trace and the person's error is reduced. By comparing the feedback from the movement and the perceptual trace, the person recognizes his/her error and can report it to the examiner and or use it through mental reinforcement.
This mental reinforcement can direct the movement to the desired goal without using the augmented feedback. In the research, it was also seen that the errorless training group which could see the simultaneous feedback of target path and its own path during the task performance, showed a significant development in identification of error.

Finally the present research specified that errorful and errorless training methods have had a significant effect on acquisition and learning of error detection in perceptual-motor skill. It also became clear that there is a significant difference between the effect of errorful and errorless training methods on error detection in tracking task and this difference was in favor of the errorless training method; i.e., the errorless training group showed greater development in error detection capability growth than the errorful training group. The general result of this research is that the lack of error in training leads to more growth in error detection than the presence of error in training. This result is in accord with Adams closed loop theory that considers the presence of error unsuitable for learning and believes that a person should always be given feedback to prevent error occurrence. The results of this research can be used in people's educating and learning of skills, especially in skills which require error detection and correction.

\section{REFERENCES}

1. Abbas Zade, Taheri, Heirani, Yousefi. Effect of giving augmented feedback methods (after success and unsuccess trials) on learning and error detection capability in force production task. Research in Sport Management \& Motor Behavior, 2013; 2(10): 99-110.

2. Azrati, Heirani, Khazaeei, Abbas Zade. Effect of giving selfcontrol and domain feedback methods on learning and error detection capability in shooting skill. Seasonal Sport Science Reasearches, 2012; 1(03): 53-64.

3. Blandin Y, Proteau L. On the cognitive basis of observational learning: development of mechanisms for the detection and correction of errors. The Quarterly Journal of Experimental Psychology: Section A, 2000; 53(3): 846-67.

4. Black, C. B., Wright, D. L., Magnuson, C. E., \& Brueckner, S. Learning to detect error in movement timing using physical and observational practice. Research Quarterly for Exercise and Sport, 2005; 76(1): 28-41.

5. Briggs G, Nebes RD. Patterns of hand preference in a student population. Cortex, 1975; 11: 230-238.

6. De Werd MM, Boelen D, Rikkert MGO, Kessels RP. Errorless learning of everyday tasks in people with dementia. Clinical Interventions in Aging, 2013; 8: 1177. 
7. Feygin D, Keehner M, Tendick F. Editors. Haptic guidance: Experimental evaluation of a haptic training method for a perceptual motor skill. Haptic Interfaces for Virtual Environment and Teleoperator Systems, 2002. Proceedings 10th Symposium on HAPTICS 2002; 2002: IEEE.

8. Grindlay G. Haptic interfaces for virtual environment and teleoperator systems. Haptics symposium, 2008: IEEE.

9. Jones RS, Clare L, MacPartlin C, Murphy O. The effectiveness of trial-and-error and errorless learning in promoting the transfer of training. European Journal of Behavior Analysis, 2010; 11: 2936.

10. Maxwell JP, Masters R, Kerr E, Weedon E. The implicit benefit of learning without errors. The Quarterly Journal of Experimental Psychology: Section A, 2001; 54(4): 1049-68.

11. Ownsworth, T., Fleming, J., Tate, R., Shum, D. H., Griffin, J., Schmidt, J., ... \& Chevignard, M. Comparison of error-based and errorless learning for people with severe traumatic brain injury: study protocol for a randomized control trial. Trials, 2013: 14(1), 369.

12. Pitel AL, Perruchet $\mathrm{P}$, Vabret $\mathrm{F}$, Desgranges $\mathrm{B}$, Eustache $\mathrm{F}$, Beaunieux $H$. The advantage of errorless learning for the acquisition of new concepts' labels in alcoholics. Psychological Medicine, 2010; 40(03): 497-502.
13. Rahmaninia F. Basics and application of motor learning. Tehran, Iran: Bamdad; 2003.

14. Sabzi. A.Mohammadi. S. Effect of self- control feedback and decreasing frequency of KR on error detection capability. Sport Science Review, 2010; 6(12): 1-10.

15. Schmidt RA, Lee T. Motor Control and Learning, 5E: Human kinetics; 2005.

16. Schmitz X, Lejeune C, Vervecken N, Meulemans T. Efficacy of errorless learning in the acquisition of a new procedural skill in Alzheimer's disease. In Annual meeting of the Belgian Association for Psychological Science (BAPS), 2011.

17. Shafizade, Bahram. Effect of auditary pattern and feedback on error detection capability and learning of timing task. Research on Sport Science, 2005; 3(8): 35-51.

18. Shayan, Huminian, Abedini, Fazel Kalkhuran. Interactional effect of modeling ( expert and self- model) and type of feedback on learning and performance of darting skill. Motor development and learning, 2013; 10: 123-142.

19. Williams, A. Mark, Nicola J. Hodges. Practice, instruction and skill acquisition in soccer: Challenging tradition. Journal of Sports Sciences 2005; 23(6): 637-650. 